\title{
Perfecting probes
}

\author{
Identifying and increasing access to the highest quality chemical probes will ensure their prominent position in the \\ biological and drug discovery toolboxes.
}

$S^{n}$ mall molecules have long had a place as tools for interrogating biological systems - for instance, colchicine was first used to investigate cell division more than 50 years ago. Over the last few decades, chemical biologists have led a focused effort to identify chemical probes spanning targets across the breadth of the genome. In comparison to genetic approaches, small molecules offer dynamic, reversible and tunable perturbations of biomolecular functions or interactions, while at the same time serving as potential leads for drug development. Despite the many advantages of chemical probes, achieving the necessary level of potency and selectivity for cellular and in vivo experiments can be a significant challenge. Carefully selecting chemical probes-and using them with the appropriate degree of caution-is essential for ensuring the rigor of resulting biological or therapeutic conclusions. As our collective experience grows, what have we learned about using chemical probes?

Chemical probe discovery has been prominently supported by the US National Institutes of Health (NIH) Molecular Libraries and Imaging (MLI) initiative, which funds the identification of small-molecule tools to "accelerate the translation of the genome sequence into biological and therapeutic insights" (Science 306, 1138-1139, 2004). Beginning in 2004, as part of the four-year initial phase of the MLI, the NIH funded the creation of ten high-throughput screening centers across the United States. Each of these centers conducted a wide range of high-throughput screens, deposited the resulting assay data in a freely available database (PubChem) and nominated chemical probes for community use. Although general guidelines for probe quality were established by the MLI, the screening centers had significant latitude in determining what constituted a 'good' probe for a particular target. To evaluate this initial phase of the MLI, Oprea et al. (p. 441) sought to assess the 64 chemical probes that were collectively nominated by the 10 screening centers. As there are no widely accepted empirical metrics for probes, 11 medicinal chemistry and drug discovery experts (the 'crowdsourced group') were asked to score, in a blinded experiment, their 'confidence' in each probe. The analysis suggests that the pilot phase of the MLI has been reasonably successful in generating quality probes, with 48 of the 64 probes being judged 'medium' or 'high' confidence. However, there were also a few nominated compounds that were not considered likely to make high-quality probes. As the next phase of the MLI begins, we encourage the screening centers to consider this and other community input in their continuing effort to implement the most rigorous and transparent probe-vetting process possible.

What constitutes a high-quality probe? Potency and selectivity are clearly critical pieces of information-but determining these parameters in vitro is only a first step. No small molecule is completely selective. As a result, ensuring the accuracy of biological or therapeutic conclusions requires verifying that a cellular or in vivo phenotype is caused by 'on-target' rather than 'off-target' effects. Though there is no single experiment to definitively make this connection, there are many types of data that can provide confidence that a compound is acting on the intended target-for instance, comparing the cellular phenotype of a chemical and genetic perturbation or inhibiting a desired target in vivo with structurally unrelated compounds. For manuscripts using chemical tools to probe biology in cells or in vivo, we (and referees) explicitly look for strong evidence that compounds are eliciting responses through the expected target. Although addressing the question of specificity is an important challenge when working with chemical tools, the potential advances in biological insights—often insights that could not be gained by genetic approaches-are substantial.

Beyond these scientific challenges, obtaining compounds can be an equally important barrier to working with chemical tools. How can access to high-quality probes be increased? Edwards et al. (p. 436) propose freely distributing compounds from drug discovery efforts, where high-quality probes are often generated but may not ever be publicly disclosed. The authors argue that the proprietary nature of the early stages of drug discovery, which encourages secrecy and duplication of effort, works to the detriment of basic biological researchand also to the detriment of the biopharmaceutical industry. Based on their recently created consortium, which includes the Structural Genomics Consortium (SGC), the NIH Chemical Genomics Center and GlaxoSmithKline among others, Edwards et al. propose conducting early drug discovery and early clinical validation in open access public-private partnerships. The SGC-led consortium will initially focus on identifying - and broadly distributing — chemical probes of epigenetic enzymes. Efforts such as this and the MLI, which identify compounds in a 'pre-competitive' environment, are providing scientists (both those focused on drug discovery and those focused on advancing our understanding of biology) with a much needed source of chemical probes.

Chemical probes are just one area in which chemical biologists are contributing innovative tools and approaches to drug discovery. To highlight some of these advances, the $3^{\text {rd }}$ Nature Chemical Biology Symposium (http://www.nature.com/natureconferences/ nchembio2009/), being held September 19 and 20 in Cambridge, Massachusetts, will focus on 'Chemical Biology in Drug Discovery'. Co-organized with Paul Workman, Guilio Superti-Furga and Brian Shoichet, the symposium will include sessions on cell-based screening and target deconvolution, targeting pathways and systems, expanding chemical space and expanding druggable targets. We hope to see you there, where we will undoubtedly hear about some of latest exciting developments in chemical probes. 